Int. J. Environ. Res. Public Health 2011, 8, 2895-2906; doi:10.3390/ijerph8072895

OPEN ACCESS

International Journal of

Environmental Research and

Public Health

ISSN 1660-4601

www.mdpi.com/journal/ijerph

Review

\title{
Alcohol Consumption and Oxidative DNA Damage
}

\section{Takeshi Hirano}

Department of Life and Environment Engineering, Faculty of Environmental Engineering, The University of Kitakyushu, 1-1 Hibikino, Wakamatsu-ku, Kitakyushu, Fukuoka 808-0135, Japan; E-Mail: t-hirano@kitakyu-u.ac.jp; Tel.: +81-93-695-3206; Fax: +81-93-695-3299

Received: 1 June 2011; in revised form: 5 July 2011 / Accepted: 6 July 2011 /

Published: 14 July 2011

\begin{abstract}
To examine the effects of alcohol consumption on cancer risk, we measured oxidative DNA damage and its repair activity in the livers and esophagi of rats fed with ethanol. Using our previously designed protocol for feeding rats with a high concentration of ethanol, we examined the effects of ethanol consumption on 8-oxo-Gua generation and repair activity in the livers and esophagi of rats. We found that a high concentration of ethanol accompanied with a vitamin-depleted diet increased 8-oxo-Gua and its repair activity. 8-Oxo-Gua is known to induce point mutations, leading to carcinogenesis. Therefore, these results suggested that a high concentration of ethanol and an irregular diet increased liver and esophageal cancer risk. On the other hand, we showed that a low concentration of ethanol decreased 8-oxo-Gua and its repair activity in the livers of mice treated with a carcinogen. Taken together, the effects of ethanol consumption on cancer risk depend on the ethanol concentration and the diet pattern.
\end{abstract}

Keywords: alcohol; 7,8-dihydro-8-oxoguanine; 8-oxoguanine DNA glycosylase 1; reactive oxygen species; vitamin-depleted diet

\section{Introduction}

An association between chronic ethanol consumption and cancer risk has been shown by epidemiological studies. Recent studies have revealed that alcohol consumption was associated with an increase in breast cancer incidence in women [1,2], esophageal cancer [3], and colorectal cancer [4,5]. Ethanol is normally metabolized to acetaldehyde, by oxidative transfer of the hydrogen of the ethanol 
by alcohol dehydrogenase $(\mathrm{ADH})$ to nicotinamide dinucleotide $\left(\mathrm{NAD}^{+}\right)$, which is reduced to $\mathrm{NADH}$, and by the microsomal ethanol oxidation system (MEOS). Xanthine oxidase oxidizes the acetaldehyde formed by ethanol metabolism and generates reactive oxygen species (ROS). Therefore, ethanol is capable of generating ROS during its metabolism. ROS are highly reactive, because they possess an unpaired electron. It is well known that ethanol increases the production of superoxide anions and hydroxyl radicals, which react rapidly with biological materials, causing oxidative damage in living organisms [6].

Long-term alcohol consumption is a major risk factor for liver disease in humans. The consumption of large amounts of ethanol over a long period can lead to liver cirrhosis and dysfunction. Liver dysfunction can also inhibit the detoxification of carcinogenic compounds that are ingested. By generating ROS, ethanol may affect the nutritional status, liver function, immune function, and other body functions, and may influence the risks for various types of cancer.

It is well known that ROS cause damage to DNA. Among the various forms of oxidative DNA damage, 7,8-dihydro-8-oxoguanine (8-oxoguanine, 8-oxo-Gua) is a major form and a useful marker of cellular oxidative stress [7]. Studies of oxidative DNA damage should be helpful in elucidating the mechanisms of cancer induction by alcohol consumption. Previously, we designed a protocol for feeding rats with a high concentration of ethanol and a vitamin-depleted diet, to induce liver fibrosis [8]. This protocol simulated the drinking and eating patterns of heavy alcohol drinkers, who tend to eat unbalanced meals at irregular intervals. Additionally, most of the heavy drinkers had started drinking when they were less than 20 years old. Based on this information, we fed young (3-week-old) rats an initial concentration of $12 \%$ ethanol and increased the concentration up to $70 \%$ with a vitamin-depleted diet (autoclaved diet, AD) or a normal diet (ND) (Figure 1). AD was prepared by autoclaving ND for $30 \mathrm{~min}$ at $121{ }^{\circ} \mathrm{C}$. The nutritional value of the diet is shown in Table 1 . The reason why the ethanol concentration was increased by $3 \% /$ week is that rats need training to drink a high concentration of ethanol; otherwise, they refuse to drink $70 \%$ ethanol. By using this protocol, we observed increases in the 8-oxo-Gua levels and its repair activity in the livers of rats after long-term alcohol- and AD-feeding [6]. In addition, we found increases in the 8-oxo-Gua levels and its repair activity in the esophagi of rats given long-term ethanol and $\mathrm{AD}$ [9]. In this article, we will describe the association between alcohol consumption and the generation of 8-oxo-Gua, by summarizing our previous work $[6,9,10]$.

Table 1. Nutrition value of the diet.

\begin{tabular}{lcl}
\hline Ingredient & Normal diet & Autoclaved diet \\
\hline Vitamin $\mathrm{B}_{1}$ & $1.73 \mathrm{mg}$ & $0.35 \mathrm{mg}(20 \%)$ \\
Vitamin $\mathrm{C}$ & $19.0 \mathrm{mg}$ & $9.0 \mathrm{mg}(47 \%)$ \\
Retinol & $0.36 \mathrm{mg}$ & $0.19 \mathrm{mg}(53 \%)$ \\
Vitamin $\mathrm{B}_{12}$ & $4.9 \mu \mathrm{g}$ & $2.6 \mu \mathrm{g}(53 \%)$ \\
Inositol & $1.63 \mathrm{mg}$ & $109 \mathrm{mg}(67 \%)$ \\
Pantoic acid & $2.85 \mathrm{mg}$ & $2.21 \mathrm{mg}(78 \%)$ \\
\hline
\end{tabular}


Table 1. Cont.

\begin{tabular}{lcl}
\hline Ingredient & Normal diet & Autoclaved diet \\
\hline Vitamin $\mathrm{B}_{6}$ & $1.26 \mathrm{mg}$ & $0.99 \mathrm{mg}(79 \%)$ \\
Niacin & $15.9 \mathrm{mg}$ & $14.0 \mathrm{mg}(88 \%)$ \\
Vitamin $\mathrm{B}_{2}$ & $1.37 \mathrm{mg}$ & $1.21 \mathrm{mg}(88 \%)$ \\
Vitamin E & $11.0 \mathrm{mg}$ & $10.0 \mathrm{mg}(91 \%)$ \\
Folic acid & $0.15 \mathrm{mg}$ & $0.15 \mathrm{mg}(100 \%)$ \\
Biotin & $47.8 \mu \mathrm{g}$ & $48.1 \mu \mathrm{g}(101 \%)$ \\
Choline & $250 \mathrm{mg}$ & $260 \mathrm{mg}(104 \%)$ \\
\hline
\end{tabular}

$\%$ of normal diet. These data were generated in 1984 and provided by Clea Japan, Inc.

Figure 1. Experimental protocol for ethanol administration. The ethanol concentration was increased from 12 to $70 \%$ over a 20 -week period. Once the concentration reached $50 \%$, $\mathrm{ND}$ and $\mathrm{AD}$ were given every other week. ND: normal diet, AD: autoclaved diet.

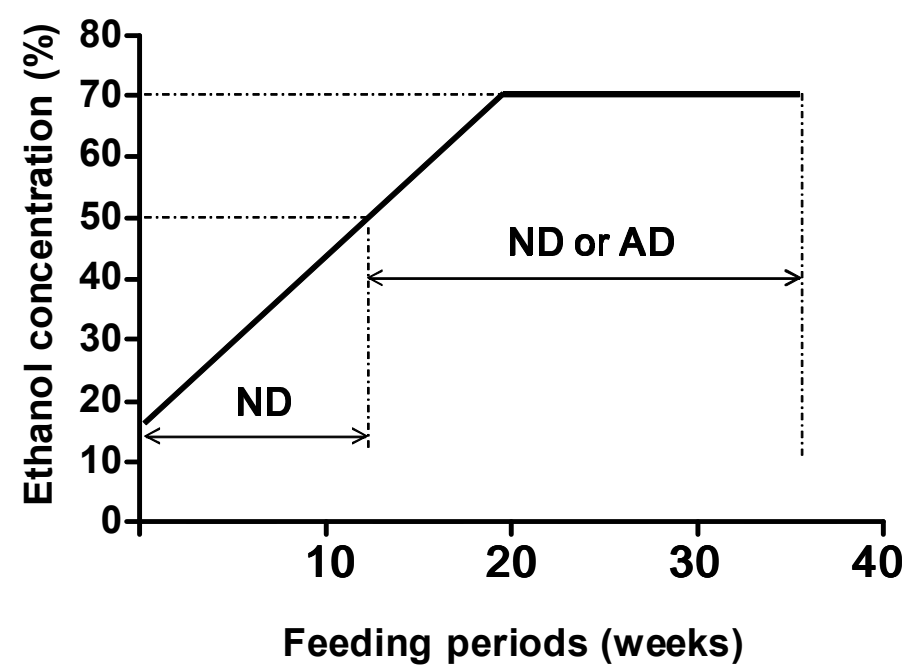

\section{8-Oxoguanine}

8-Oxo-Gua is a mutagenic lesion formed spontaneously in the genomic DNA of aerobic organisms (Figure 2A) and by the actions of exogenous factors, such as ionizing radiation, chemical pollutants, metals, food, and bacteria. Although 8-oxo-Gua is not necessarily the most abundant form of oxidative DNA damage, it has been the most extensively studied, because it can be quantitated with high sensitivity by high performance liquid chromatography coupled with electrochemical detection (HPLC-ECD), and is quite easily measured in laboratories [11,12]. We have studied the relationship between the 8-oxo-Gua levels and health-related factors, such as chemical agents [13-15], $\gamma$-irradiation [16], aging [17-19], and physical exercise [20,21]. 8-Oxo-Gua is thought to be responsible for carcinogenesis, because it induces the GC-to-TA transversion type point mutation in DNA in vitro [22,23]. In fact, several reports have supported this premise, based on experiments with cultured cells and with animals [13-15,24,25]. Therefore, analyses of 8-oxo-Gua generation are useful to understand the detailed mechanisms of carcinogenesis. 


\section{8-Oxoguanine Repair System}

DNA repair systems maintain the integrity of the mammalian genome by removing DNA damage, reducing the mutation frequency of cancer-related genes, minimizing replication errors, and curtailing deleterious rearrangements arising via aberrant recombination [26]. If 8-oxo-Gua is not repaired, then it leads to the accumulation of GC-to-TA point mutations (Figure 2B), suggesting that 8-oxo-Gua may play a key role in carcinogenesis [22,27-29]. From the earliest stage of 8-oxo-Gua research, repair systems for 8-oxo-Gua were predicted to exist [30].

Since 8-oxo-Gua was reported in 1984 [7], many researchers have tried to detect and clone its repair enzymes. An endonuclease nicking assay indicated the existence of a repair system for 8-oxo-Gua in a wide variety of living organisms, including bacteria, yeast, mammals, and even plants. The first information was obtained from studies of the enzyme formamidopyrimidine DNA glycosylase (Fpg or MutM), which excises 8-oxo-Gua, 2,6-diamino-4-hydroxy-5-formamidopyrimidine (FapyGua), and 4,6-diamino-5-formamidopyrimidine (FapyAde) from the DNA of Escherichia coli [31]. In 1996, an Fpg homologue was identified in Saccharomyces cerevisiae [32,33]. This was the first report for 8-oxoguanine DNA glycosylase 1 (OGG1). In the following year, mammalian (human and other mammals) homologues of OGG1 were identified and cloned [34-40]. In terms of the gene stability associated with DNA damage and its repair systems, the prevalence of 8-oxo-Gua repair enzymes highlights the primary importance of 8-oxo-Gua and OGG1 in most living organisms.

Figure 2. (A) Structure of 8-oxo-Gua. 8-Oxo-Gua is formed by the hydroxylation of guanine at the C-8 position; and (B) Mechanism of GC-to-TA point mutation induction. OGG1 is 8-oxoguanine DNA glycosylase, which removes 8-oxo-Gua generated in DNA. G: 8-oxo-Gua.

(A)<smiles>Cn1c(=O)[nH]c2c(=O)[nH]c(N)nc21</smiles>

\section{7, 8-dihydro-8-oxoguanine (8-oxo-Gua)}

(B)

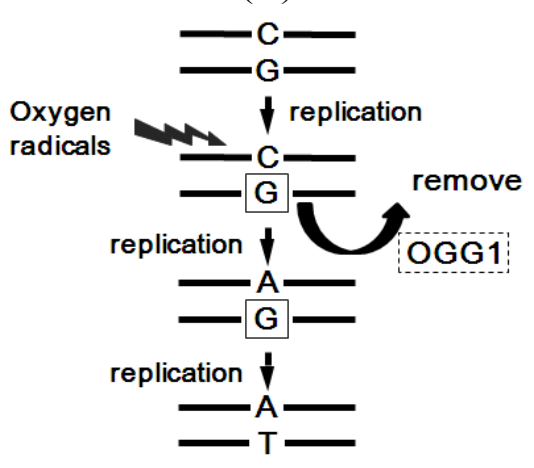

OGG1 removes 8-oxo-Gua from damaged DNA in the base excision repair (BER) process, to prevent the GC-to-TA transversion type of point mutation (Figure 2B) [34,35,41]. The GC-to-TA point mutation is commonly observed in the tumor suppressor $p 53$ gene in human cancers, such as lung cancer [42-45]. In addition, Hussain and Harris reported that $33 \%$ of the somatic $p 16^{I N K 4}$ mutations in human cancers were GC-to-TA transversions [46]. Moreover, mutations of the OGG1 gene in human lung cancer have also been reported [36,47-49]. It is reasonable to assume that the $O G G 1$ mutation may be associated with the GC-to-TA point mutations that occur in the tumor-associated genes of living organisms. OGG1 is known to have glycosylase activity for other lesions, in addition to 
8-oxo-Gua. Jensen et al. reported that OGG1 also incised 8-oxoadenine opposite cytosine in nuclei and mitochondria [50]. This evidence suggests that OGG1 plays a fundamental role in maintaining DNA stability.

As a line of defense against mutagenesis or carcinogenesis, a repair system, called the GO system, acts in response to 8-oxo-Gua generation [51]. The GO system contains three main enzymes: OGG1, MutY homolog (MUTYH), and MutT homolog 1 (MTH1). MUTYH excises adenine incorporated opposite 8-oxo-Gua, as a mis-match repair enzyme [52]. MTH1 is a clearance enzyme that acts as an oxidized purine nucleoside triphosphatase for 8-oxo-GTP in the nucleotide pool [53]. These three enzymes coordinately prevent 8-oxo-Gua accumulation and, thus, point mutations in DNA. It is noteworthy that the measured level of 8-oxo-Gua depends on the balance between its generation and repair activity. Therefore, to understand 8-oxo-Gua-associated carcinogenesis, studies on both the 8-oxo-Gua levels and its repair ability are required. Indeed, many studies suggested that an imbalance between them might be involved in carcinogenesis.

\section{Alcohol and 8-Oxoguanine in Liver DNA}

We analyzed 8-oxo-Gua generation and repair activity in the livers of rats subjected to long-term consumption of ethanol and AD [6]. Male Sprague-Dawley rats (3-week-old) were fed an ethanol beverage whose concentration was increased by $3 \%$ every week, starting at $12 \%$ and progressing up to $70 \%$ (Figure 1). When the concentration reached $50 \%$, the diet of one group was changed from the ND to either $\mathrm{AD}$ or ND. At the feeding periods of 18, 20, and 30 weeks (ethanol concentrations were 66, 70 , and $70 \%$, respectively), the rats were sacrificed. The livers were immediately excised and the levels of 8-oxo-Gua and its repair activity were measured. The 8-oxo-Gua levels in the livers of ethanol-fed rats at the feeding periods of 18 and 20 weeks showed no change, in comparison to those in the livers of the control rats. The 8-oxo-Gua repair activity also showed no difference at the same feeding periods. However, at the feeding period of 30 weeks, both the 8-oxo-Gua level in the livers of the ethanol-fed rats and its repair activity were increased, as compared to the levels in the control rats (Figure 3). These results indicated that the long-term consumption of a high concentration of alcohol might increase the risks of developing cancer in the liver, derived from oxidative DNA damage.

\section{Alcohol and 8-Oxoguanine in Esophageal DNA}

We analyzed the 8-oxo-Gua generation level and repair activity in the esophagus, in rats subjected to long-term consumption of ethanol and AD [9]. Male Sprague-Dawley rats (3-week-old) were fed an ethanol beverage whose concentration was increased from 12 to $70 \%$ over 20 weeks (Figure 1). When the concentration reached $50 \%$, the diet of one group was changed from the ND to AD. At the feeding periods of 20,25,30, and 35 weeks, the rats were sacrificed and the 8-oxo-Gua levels and repair activities within the esophagi were measured. After 30 weeks of ethanol- and AD-feeding, significant increases of 8-oxo-Gua and its repair activity were observed in the esophagi, but not in those of the ethanol- and ND-fed rats (Figure 4). This result indicates that the combined effects of long-term ethanol consumption and vitamin deficiency may be involved in inducing oxidative DNA damage in the rat esophagus. 
Figure 3. (A) 8-Oxo-Gua levels in ethanol-fed rats at 18, 20 and 30 weeks of feeding. The value of 8-oxo-Gua is expressed as 8 -oxo-dG/10 $\mathrm{dG}$; (B) 8-Oxo-Gua repair activity levels in ethanol-fed rats at 18, 20 and 30 weeks of feeding. The data are expressed as the ratios to the control. The assessment method for 8-oxo-Gua repair activity (endonuclease nicking assay) was described elsewhere [9]. Briefly, the oligonucleotide containing 8-oxo-Gua was used as a substrate for the assay. The total crude extract obtained from animal tissue was incubated with end-labeled double-stranded DNA substrates at $25^{\circ} \mathrm{C}$ for $1 \mathrm{hr}$. After ethanol precipitation, the pellet was dried, dissolved in $10 \mu \mathrm{L}$ of loading buffer and denatured by heating at $90{ }^{\circ} \mathrm{C}$ for $3 \mathrm{~min}$. A $10 \mu \mathrm{L}$ portion of the sample was applied to a $20 \%$ denaturing polyacrylamide gel for electrophoresis. 8-oxo-dG: 7,8-dihydro-8-oxoguanosine.

(A)

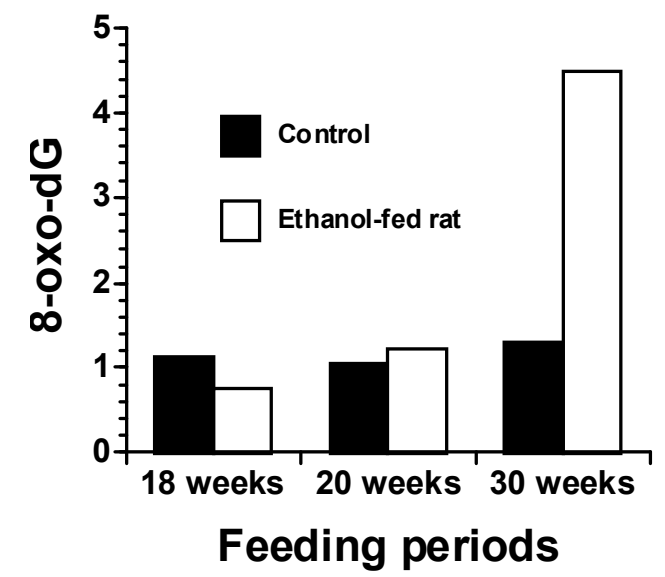

(B)

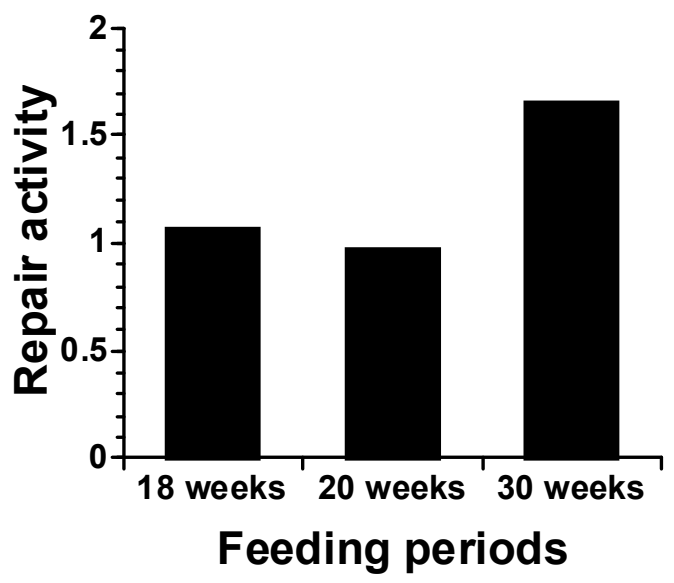

Figure 4. Autoradiogram showing a comparison of the esophageal 8-oxo-Gua repair activities in rats fed ethanol and $\mathrm{AD}$ for 30 weeks with those fed ND. Upper band, substrate DNA; lower band (arrow head), excised fragment. The assessment method for the 8-oxo-Gua repair activity (endonuclease nicking assay) was described in the legend of Figure 3. This figure was published in reference [9], Copyright Wiley-Blackwell. ND: normal diet, AD: autoclaved diet.

\section{$\mathrm{EtOH}(+), \mathrm{AD}$}

$\operatorname{EtOH}(-)$, ND

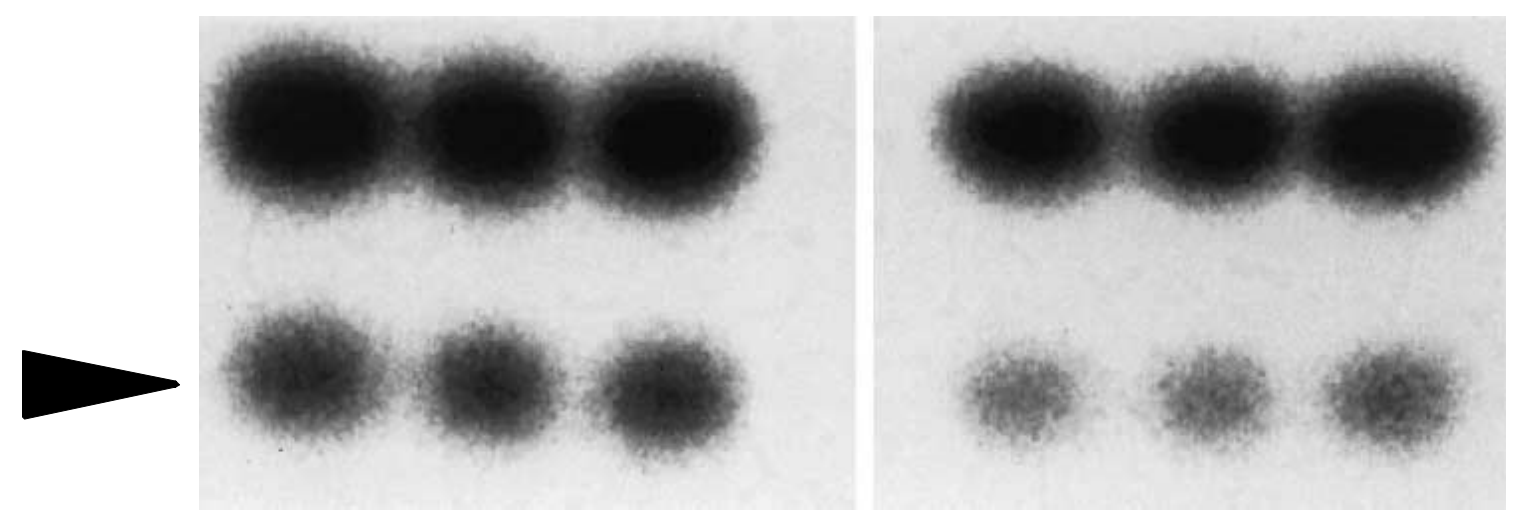




\section{Chronic Alcohol Consumption Prevents 8-Oxoguanine Accumulation in the Livers of Carcinogen-Treated Mice}

Recently, we reported the effect of alcohol consumption on 8-oxo-Gua generation in the livers of 3'-methyl-4-dimethylaminoazobenzene (3'-MeDAB)-treated mice [10]. We fed 3'-MeDAB to mice for eight months with/without $12 \%$ ethanol, and measured the 8-oxo-Gua generation level and its repair activity in the liver (Figure 5A). 3'-MeDAB is a type of amino azo dye and is highly hepato-carcinogenic [54]. The tumorigenic mechanisms are complex, and are thought to include carcinogen-DNA adduct formation [55,56] and ROS generation [57]. The results showed that 12\% ethanol intake attenuated the 8-oxo-Gua accumulation, suggesting that $12 \%$ ethanol consumption may reduce the risk of 3 '-MeDAB-induced carcinogenesis by decreasing 8-oxo-Gua accumulation (Figures 5B and C).

Figure 5. (A) The experimental protocol. A: control [ND/water] for 10 months; B: [ND/water] for the first 2 months and [0.06\% 3'-MeDAB/water] for the last 8 months; $\mathrm{C}$ : [ND/alcohol] for 10 months; D: [ND/alcohol] for the first 2 months and [0.06\% 3'-MeDAB/alcohol] for the last 8 months; E: [ND/alcohol] for the first 2 months, [0.06\% 3'-MeDAB/alcohol] for the next 4 months, and [0.06\% 3'-MeDAB/water] for the last 4 months; F: [ND/water] for the first 2 months, [3'-MeDAB/water] for the next 4 months, and [3'-MeDAB/alcohol] for the last 4 months; (B) The levels of 8-oxo-Gua in the DNA of mouse livers. The 8-oxo-Gua value is expressed as the number of 8-oxo-dG per $10^{5}$ deoxyguanosine. ${ }^{*} 1: P<0.0005$ vs. group $\mathrm{B}, P<$ 0.05 vs. group $\mathrm{E}, P<0.01$ vs. group $\mathrm{F} ; * 2: P<0.0001$ vs. group $\mathrm{C}, P<0.005$ vs. group $\mathrm{D}, P<$ 0.01 vs. group $\mathrm{E}, P<0.05$ vs. group $\mathrm{F}$; $* 3: P<0.05$ vs. group $\mathrm{E}, P<0.005$ vs. group $\mathrm{F} ; * 4: P<$ 0.05 vs. group F; and (C) 8-Oxo-Gua nicking activity in the mouse livers. The activity was calculated as the ratio of the excised fragment intensity to the total substrate (unexcised substrate intensity plus excised fragment intensity). *: $P<0.005$ vs. group A. ND: normal diet, AD: autoclaved diet, 8-oxo-dG: 7, 8-dihydro-8-oxoguanosine. This figure was published in reference [10], Copyright Elsevier.

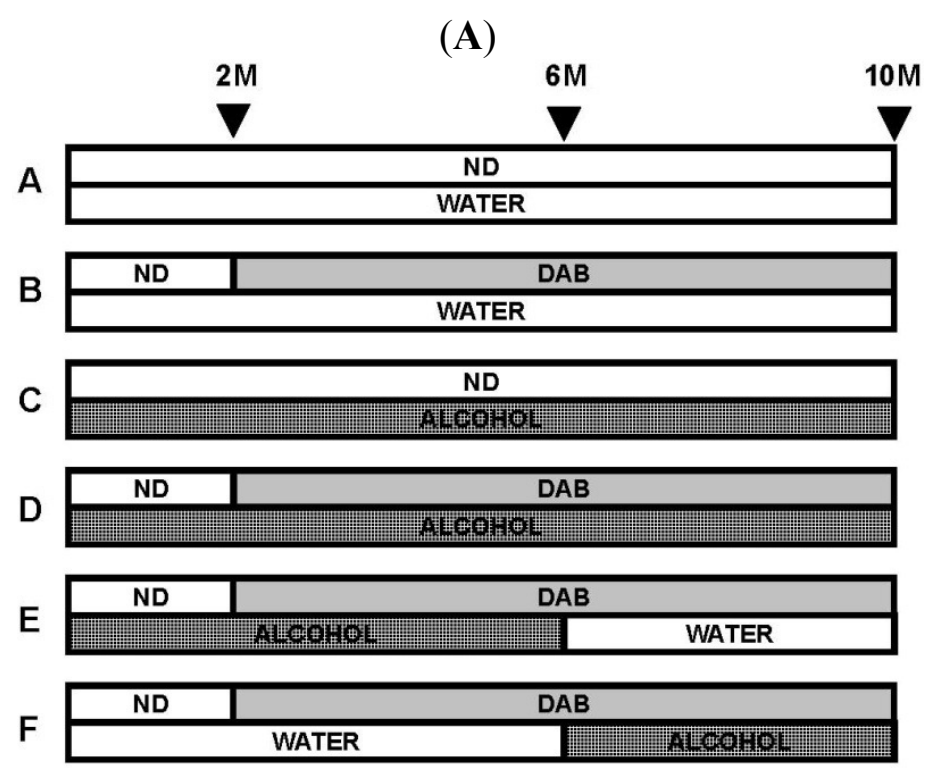


Figure 5. Cont.

(B)

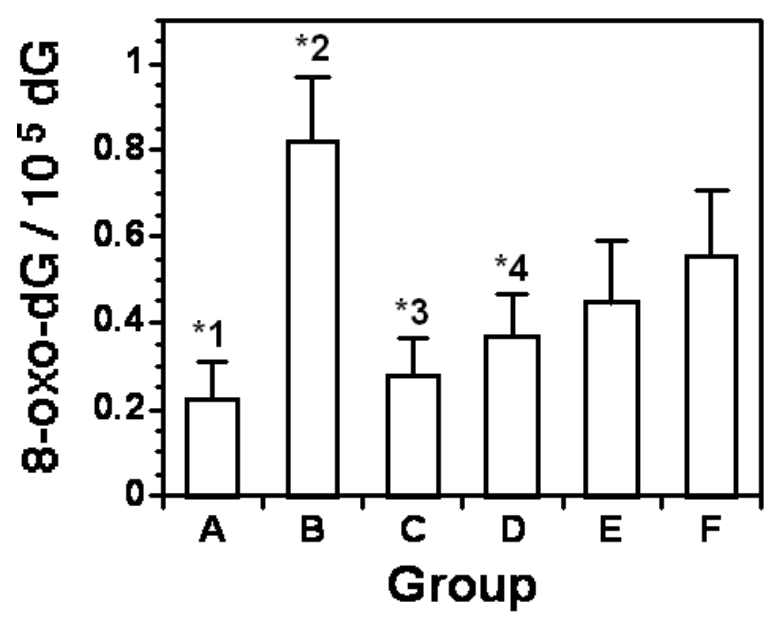

(C)

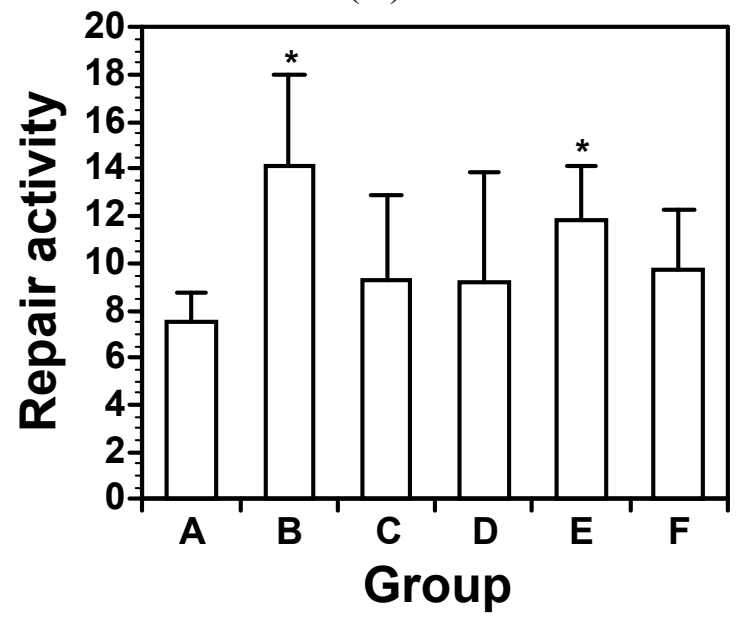

\section{Conclusions}

As shown in this article, alcohol exhibits at least two different features, in terms of carcinogenesis. One is an enhanced effect for carcinogenesis and the other is an inhibitory effect. Our study indicated that these effects of alcohol consumption on carcinogenesis may depend on the drinking pattern and the diet. To drink alcohol safely, the consumption pattern and the diet should be considered.

\section{Acknowledgements}

The authors thank David J. Hanson, guest editor of this special issue, for inviting us to write this review article, Wiley-Blackwell and Elsevier LTD for permission to reproduce the material in references [9] and [10], respectively, and Clea Japan Inc. for providing the diet data shown in Table 1.

\section{References}

1. Smith-Warner, S.A.; Spiegelman, D.; Yaun, S.S.; van den Brandt, P.A.; Folsom, A.R.; Goldbohm, A.; Graham, S.; Holmberg, L.; Howe, G.R.; Marshall, J.R.; et al. Alcohol and breast cancer in women: A pooled analysis of cohort studies. JAMA 1998, 279, 535-540.

2. Stolzenberg-Solomon, R.Z.; Chang, S.C.; Leitzmann, M.F.; Johnson, K.A.; Johnson, C.; Buys, S.S.; Hoover, R.N.; Ziegler, R.G. Folate intake, alcohol use, and postmenopausal breast cancer risk in the prostate, lung, colorectal, and ovarian cancer screening trial. Am. J. Clin. Nutr. 2006, 83, 895-904.

3. Wu, M.; Zhao, J.K.; Hu, X.S.; Wang, P.H.; Qin, Y.; Lu, Y.C.; Yang, J.; Liu, A.M.; Wu, D.L.; Zhang, Z.F.; et al. Association of smoking, alcohol drinking and dietary factors with esophageal cancer in high- and low-risk areas of Jiangsu Province, China. World J. Gastroenterol. 2006, 12, 1686-1693.

4. Klatsky, A.L.; Armstrong, M.A.; Friedman, G.D. The relations of alcoholic beverage use to colon and rectal cancer. Am. J. Epidemiol. 1998, 128, 1007-1015.

5. Ho, J.W.; Lam, T.H.; Tse, C.W.; Chiu, L.K.; Lam, H.S.; Leung, P.F.; Ng, K.C.; Ho, S.Y.; Woo, J.; Leung, S.S.; et al. Smoking, drinking and colorectal cancer in Hong Kong Chinese: A case control 
study. Int. J. Cancer 2004, 109, 587-597.

6. Hirano, T.; Homma, Y.; Kasai, H. Formation of 8-Hydroxyguanine in DNA by Aging and Oxidative Stress. In Oxidative Stress and Aging; Cutler, R.G., Packer, L., Bertran, J., Mori, A., Eds.; Birkhauser Verlag: Basel, Switzerland, 1995; pp. 69-76.

7. Kasai, H.; Nishimura, S. Hydroxylation of deoxyguanosine at the C-8 position by ascorbic acid and other reducing agents. Nucleic Acids Res. 1984, 12, 2137-2145.

8. Hirano, H.; Hirano, T.; Hirata, T.; Tamura, M.; Yamaura, T.; Hamada, T. Experimental liver fibrosis induced in rats receiving high doses of alcohol and alternating between regular and vitamin-depleted diets. Experientia 1996, 52, 710-715.

9. Asami, S.; Hirano, T.; Yamaguchi, R.; Tsurudome, Y.; Itoh, H.; Kasai, H. Increase in 8-hydroxyguanine and its repair activity in the esophagi of rats given long-term ethanol and nutrition-deficient diet. Jpn. J. Cancer Res. 2000, 91, 973-978.

10. Hirano, T.; Sakai, A.; Ootsuyama, Y.; Kasai, H. Chronic alcohol consumption prevents 8-hydroxyguanine accumulation in 3'-methyl-4-dimethylaminoazobenzene-treated mouse liver. Biochem. Biophys. Res. Commun. 2009, 387, 316-320.

11. Floyd, R.A.; Watson, J.J.; Wong, P.K.; Altmiller, D.H.; Rickard, R.C. Hydroxyl free radical adduct of deoxyguanosine: Sensitive detection and mechanisms of formation. Free Radical Res. Commun. 1986, 1, 163-172.

12. Marnett, L.J. Oxyradicals and DNA damage. Carcinogenesis 2000, 21, 361-370.

13. Hirano, T.; Yamaguchi, Y.; Kasai, H. Inhibition of 8-hydroxyguanine repair in testes after administration of cadmium chloride to GSH-depleted rats. Toxicol. Appl. Pharmacol. 1997, 147, 9-14.

14. Tsurudome, Y.; Hirano, T.; Yamato, H.; Tanaka, I.; Sagai, M.; Hirano, H.; Nagata, N.; Itoh, H.; Kasai, H. Changes in levels of 8-hydroxyguanine in DNA, its repair and OGG1 mRNA in rat lungs after intratracheal administration of diesel exhaust particles. Carcinogenesis 1999, 20, 1573-1576.

15. Hirano, T.; Higashi, K.; Sakai, A.; Tsurudome, Y.; Ootsuyama, Y.; Kido, R.; Kasai, H. Analyses of oxidative DNA damage and its repair activity in the livers of 3'-methyl-4-dimethylaminoazobenzene-treated rodents. Jpn. J. Cancer Res. 2000, 91, 681-685.

16. Mei, N.; Tamae, K.; Kunugita, N.; Hirano, T.; Kasai, H. Analysis of 8-hydroxydeoxyguanosine 5'-monophosphate (8-OH-dGMP) as a reliable marker of cellular oxidative DNA damage after gamma-irradiation. Environ. Mol. Mutagen. 2003, 41, 332-338.

17. Hirano, T.; Yamaguchi, Y.; Hirano, H.; Kasai, H. Age-associated change of 8-hydroxyguanine repair activity in cultured human fibroblasts. Biochem. Biophys. Res. Commun. 1995, 214, 1157-1162.

18. Hirano, T.; Yamaguchi, R.; Asami, S.; Iwamoto, N.; Kasai, H. 8-Hydroxyguanine levels in nuclear DNA and its repair activity in rat organs associated with age. J. Gerontol. 1996, A51, B303-B307.

19. Tsurudome, Y.; Hirano, T.; Hirata, K.; Higure, A.; Nagata, N.; Takahashi, K.; Itoh, H.; Kasai, H. Age associated increase of 8-hydroxydeoxyguanosine in human colorectal tissue DNA. J. Gerontol. 2001, 56A, B483-B485.

20. Asami, S.; Hirano, T.; Yamaguchi, R.; Tomioka, Y.; Itoh, H.; Kasai, H. Increase of a type of oxidative DNA damage, 8-hydroxyguanine, and its repair activity in human leukocytes by cigarette smoking. Cancer Res. 1996, 56, 2546-2549. 
21. Asami, S.; Hirano, T.; Yamaguchi, R.; Tsurudome, Y.; Itoh, H.; Kasai, H. Effects of forced and spontaneous exercise on 8-hydroxydeoxyguanosine levels in rat organs. Biochem. Biophys. Res. Commun. 1998, 243, 678-682.

22. Cheng, K.C.; Cahill, D.S.; Kasai, H.; Nishimura, S.; Loeb, L.A. 8-Hydroxyguanine, an abundant form of oxidative DNA damage, causes $\mathrm{G} \rightarrow \mathrm{T}$ and $\mathrm{A} \rightarrow \mathrm{C}$ substitutions. J. Biol. Chem. 1992, 267, 166-172.

23. Basu, A.K.; Loechler, E.L.; Leadon, S.A.; Essigmann, J.M. Genetic effects of thymine glycol: Site-specific mutagenesis and molecular modeling studies. Proc. Natl. Acad. Sci. USA 1989, 86, 7677-7681.

24. Yamaguchi, R.; Hirano, T.; Asami, S.; Chung, K.M.; Sugita, A.; Kasai, H. Increased 8-hydroxyguanine levels in DNA and its repair activity in rat kidney after administration of a renal carcinogen, ferric nitrilotriacetate. Carcinogenesis 1996, 17, 2419-2422.

25. Mei, N.; Kunugita, N.; Hirano, T.; Kasai, H. Acute arsenite-induced 8-hydroxyguanine is associated with inhibition of repair activity in cultured human cells. Biochem. Biophys. Res. Commun. 2002, 297, 924-930.

26. Hoeijimakers, J.H. Genome maintenance mechanisms for preventing cancer. Nature 2001, 411, 366-374.

27. Wood, M.L.; Dizdarogle, M.; Gajewski, E.; Essigmann, J.M. Mechanistic studies of ionizing radiation and oxidative mutagenesis: Genetic effects of a single 8-hydroxyguanine (7-hydro-8-oxoguanine) residue inserted at a unique site in a viral genome. Biochemistry 1990, 29, 7024-7032.

28. Shibutani, S.; Takeshita, M.; Grollman, A.P. Insertion of specific bases during DNA synthesis past the oxidation-damaged base 8-oxodG. Nature 1991, 349, 431-434.

29. Kamiya, H.; Miura, K.; Ishikawa, H.; Inoue, H.; Nishimura, S.; Ohtsuka, E. c-Ha-ras containing 8-hydroxyguanine at codon 12 induces point mutations at the modified and adjacent positions. Cancer Res. 1992, 52, 3483-3485.

30. Kasai, H.; Crain, P.F.; Kuchino, Y.; Nishimura, S.; Ootsuyama, A.; Tanooka, H. Formation of 8-hydroxyguanine moiety in cellular DNA by agents producing oxygen radicals and evidence for its repair. Carcinogenesis 1986, 11, 1849-1851.

31. Boiteux, S.; O’Connor, T.R.; Lederer, F.; Gouyette, A.; Laval, J. Homogeneous Escherichia coli FPG protein. J. Biol. Chem. 1990, 265, 3916-3922.

32. Auffret van der Kemp, P.; Thomas, D.; Barbey, R.; De Oliveira, R.; Boiteux, S. Cloning and expression in Escherichia coli of the OGG1 gene of Saccharomyces cerevisiae, which codes for a DNA glycosylase that excises 7,8-dihydro-8-oxoguanine and 2,6-diamino-4-hydroxy-5- $N$-methyl-formamidopyrimidine. Proc. Natl. Acad. Sci. USA 1996, 93, 5197-5202.

33. Nash, H.M.; Bruner, S.D.; Schärer, O.D.; Kawate, T.; Addona, T.A.; Sponner, E.; Lane, W.S.; Verdine, G.I. Cloning of a yeast 8-oxoguanine DNA glycosylase reveals the existence of a base-excision DNA-repair protein superfamily. Curr. Biol. 1996, 6, 968-980.

34. Rosenquist, T.A.; Zharkov, D.O.; Grollman, A.P. Cloning and characterization of a mammalian 8-oxoguanine DNA glycosylase. Proc. Natl. Acad. Sci. USA 1997, 94, 7429-7434. 
35. Radicella, J.P.; Dherin, C.; Desmaze, C.; Fox, M.S.; Boiteux, S. Cloning and characterization of hOGG1, a human homolog of OGG1 gene of Saccharomyces cerevisiae. Proc. Natl. Acad. Sci. USA 1997, 94, 8010-8015.

36. Lu, R.; Nash, H.M.; Verdine, G.L. A mammalian DNA repair enzyme that excises oxidatively damaged guanines maps to a locus frequently lost in lung cancer. Curr. Biol. 1997, 7, 397-407.

37. Roldán-Arjona, T.; Wei, Y.F.; Carter, K.C.; Klungland, A.; Anselmino, C.; Wang, R.P.; Augustus, M.; Lindahl, T. Molecular cloning and functional expression of a human cDNA encoding the antimutator enzyme 8-hydroxyguanine-DNA glycosylase. Proc. Natl. Acad. Sci. USA 1997, 94, 8016-8015.

38. Bjoräs, M.; Luna, L.; Johnson, B.; Hoff, E.; Haug, T.; Rongnes, T.; Seeberg, E. Opposite base-dependent reactions of a human base excision repair enzyme on DNA containing 7,8-dihydro-8-oxoguanine and abasic sites. EMBO J. 1997, 16, 6314-6322.

39. Arai, K.; Morishita, K.; Shinmura, K.; Kohno, T.; Kim, S.R.; Nohmi, T.; Taniwaki, M.; Ohwada, S.; Yokota, J. Cloning of a human homolog of the yeast OGG1 gene that is involved in the repair of oxidative DNA damage. Oncogene 1997, 14, 2857-2861.

40. Aburatani, H.; Hippo, Y.; Ishida, T.; Takashima, R.; Matsuba, C.; Kodama, T.; Takao, M.; Yasui, A.; Yamamoto, K.; Asano, M.; et al. Cloning and characterization of mammalian 8-hydroxy- guanine-specific DNA glycosylase/apurinic, a pyrimidinic lyase, a functional mutM homologue. Cancer Res. 1997, 57, 2151-2156.

41. Tani, M.; Shinmura, K.; Kohno, T.; Shiroishi, T.; Wakana, S.; Kim, S.R.; Nohmi, T.; Kasai, H.; Takenoshita, S.; Nagamachi, Y.; et al. Genomic structure and chromosomal localization of the mouse $O g g 1$ gene that is involved in the repair of 8-hydroxyguanine in DNA damage. Mamm. Genome 1989, 9, 32-37.

42. Lehman, T.A.; Bennett, W.P.; Metcalf, R.A.; Welsh, J.A.; Ecker, J.; Modali, R.V.; Ullrich, S.; Romano, J.W.; Appella, E.; Testa, J.R.; et al. p53 mutations, ras mutations and p53-heat shock 70 protein complexes in human lung carcinoma cell lines. Cancer Res. 1991, 51, 4090-4096.

43. Iggo, R.; Gatter, K.; Bartek, J.; Lane, D.; Harris, A.L. Increased expression of mutant forms of p53 oncogene in primary lung cancer. Lancet 1990, 335, 675-679.

44. Takahashi, T.; Nau, M.M.; Chiba, I.; Birrer, M.J.; Rosenberg, R.K.; Vinocour, M.; Levitt, M.; Pass, H.; Gazdar, A.F.; Minna, J.D. P53: A frequent target for genetic abnormalities in lung cancer. Science 1989, 246, 491-494.

45. D’Amico, D.; Carbone, D.; Mitsudomi, T.; Nau, M.; Fedorko, J.; Russell, E.; Johnson, B.; Buchhagen, D.; Bodner, S.; Phelps, R. High frequency of somatically acquired p53 mutations in small-cell lung cancer cell lines and tumors. Oncogene 1992, 7, 339-346.

46. Hussain, S.P.; Harris, C.C. Molecular epidemiology of human cancer: Contribution of mutation spectra studies of tumor suppressor genes. Cancer Res. 1998, 58, 4023-4037.

47. Chevillard, S.; Radicella, J.P.; Levalois, C.; Lebeau, J.; Poupon, M.F.; Oudard, S.; Dutrillaux, B.; Boiteux, S. Mutations in OGG1, a gene involved in the repair of oxidative DNA damage, are found in human lung and kidney tumours. Oncogene 1998, 16, 3083-3086.

48. Sugimura, H.; Kohno, T.; Wakai, K.; Nagura, K.; Genka, K.; Igarashi, H.; Morris, B.J.; Baba, S.; Ohno, Y.; Gao, C.; et al. hOGG1 Ser326Cys polymorphism and lung cancer susceptibility. Cancer Epidemiol. Biomarkers Prev. 1999, 8, 669-674. 
49. Wikman, H.; Risch, A.; Klimek, F.; Schmezer, P.; Spiegelhalder, B.; Dienemann, H.; Kayser, K.; Schulz, V.; Drings, P.; Bartsch, H. hOGG1 polymorphism and loss of heterozygosity (LOH): Significance for lung cancer susceptibility in a Caucasian population. Int. J. Cancer 2000, 88, 932-937.

50. Jensen, A.; Calvayrac, G.; Karahalil, B.; Bohr, V.A.; Stevnsner, T. Mammalian 8-oxoguanine DNA glycosylase 1 incises 8-oxoadenine opposite cytosine in nuclei and mitochondria, while a different glycosylase incises 8-oxoadenine opposite guanine in nuclei. J. Biol. Chem. 2003, 278, 19541-19548.

51. Hirano, T. Repair system of 7,8-dihydro-8-oxoguanine as a defense line against carcinogenesis. J. Radiat. Res. 2008, 49, 329-340.

52. Lu, A.L.; Fawcett, W.P. Characterization of the recombinant MutY homolog, an adenine DNA glycosylase, from yeast Schizosaccharomyces pombe. J. Biol. Chem. 1998, 273, 25098-25105.

53. Kakuma, T.; Nishida, J.; Tsuzuki, T.; Sekiguchi, M. Mouse MTH1 protein with 8-oxo-7,8-dihydro-2'-deoxyguanosine 5'-triphosphatase activity that prevents transversion mutation. J. Biol. Chem. 1995, 270, 25942-25948.

54. Labuc, G.E.; Blunck, J.M. Metabolic activation of the hepatocarcinogen 3'-methyl-4-dimethyl-aminoazobenzene by a rat liver cell-free-system. Biochem. Pharmacol. 1979, 28, 2367-2373.

55. Tarpley, W.G.; Miller, J.A.; Miller, E.C. Adducts from the reaction of $\mathrm{N}$-benzoyloxy- $\mathrm{N}$-methyl-4-aminoazobenzene with deoxyguanosine or DNA in vitro and from hepatic DNA of mice treated with $\mathrm{N}$-methyl- or $\mathrm{N}, \mathrm{N}$-dimethyl-4-aminoazobenzene. Cancer Res. 1980, 40, 2493-2499.

56. Tullis, D.L.; Dooley, K.L.; Miller, D.W.; Baetcke, K.P.; Kadlubar, F.F. Characterization and properties of the DNA adducts formed from $N$-methyl-4-aminoazobenzene in rats during a carcinogenic treatment regimen. Carcinogenesis 1987, 8, 577-583.

57. Duchesne, J.; Goutier, R.; Maréchal, R.; van de Vorst, A. Free radical evolution in rat liver following the ingestion of DAB. Phys. Med. Biol. 1975, 20, 305-309.

(C) 2011 by the authors; licensee MDPI, Basel, Switzerland. This article is an open access article distributed under the terms and conditions of the Creative Commons Attribution license (http://creativecommons.org/licenses/by/3.0/). 